\title{
Promoter methylation profiles of tumor suppressor genes in intrahepatic and extrahepatic cholangiocarcinoma
}

\author{
Bin Yang ${ }^{1}$, Michael G House ${ }^{2}$, Mingzhou Guo², James G Herman² and Douglas P Clark ${ }^{1}$ \\ ${ }^{1}$ Department of Pathology and ${ }^{2}$ The Sidney Kimmel Comprehensive Cancer Center, The Johns Hopkins \\ Medical Institutions, Baltimore, MD, USA
}

\begin{abstract}
Recent studies indicate that tumor suppressor genes can be epigenetically silenced through promoter hypermethylation. To further understand epigenetic alterations in cholangiocarcinoma, we have studied the methylation profiles of 12 candidate tumor suppressor genes (APC, E-cadherin/CDH1, MGMT, RASSF1A, GSTP, $R A R-\beta, p 14^{A R F}, p 15^{I N K 4 b}, p 16^{I N K 4 a}, p 73, h M L H 1$ and DAPK) in 72 cases of cholangiocarcinoma, including equal number cases of intrahepatic cholangiocarcinoma and extrahepatic cholangiocarcinoma. A total of 10 cases of benign biliary epithelia were included as controls. The methylation status of tumor suppressor genes was analyzed using methylation-specific PCR. We found that $85 \%$ of all cholangiocarcinomas had methylation of at least one tumor suppressor gene. The frequency of tumor suppressor gene methylation in cholangiocarcinoma was: RASSF1A $(65 \%)$, p15 INK4b $(50 \%)$, p16 INK4a $(50 \%)$, APC $(46 \%)$, E-cadherin/CDH1 $(43 \%)$, p14 $4^{A R F}(38 \%)$, p73 (36\%), MGMT (33\%), hMHL1 (25\%), GSTP (14\%), RAR- $\beta(14 \%)$ and DAPK (3\%). Although single tumor suppressor gene methylation can be seen in benign biliary epithelium, methylation of multiple tumor suppressor genes is only seen in cholangiocarcinoma. About $70 \%(50 / 72)$ of the cholangiocarcinomas had three or more tumor suppressor genes methylated and $52 \%(38 / 72)$ of cases had four or more tumor suppressor genes methylated. Concerted methylation of multiple tumor suppressor genes was closely associated with methylation of RASSF1A, p16 and/or hMHL1. Methylation of RASSF1A was more common in extrahepatic cholangiocarcinoma than intrahepatic cholangiocarcinoma (83 vs $47 \%, P=0.003)$ while GSTP was more frequently seen in intrahepatic compared to extrahepatic cholangiocarcinoma (31 vs $6 \%, P=0.012)$. Our study indicates that methylation of promoter CpG islands of tumor suppressor genes is a common epigenetic event in cholangiocarcinoma. Based on distinct methylation profiles, intrahepatic cholangiocarcinoma and extrahepatic cholangiocarcinoma are two closely related but biologically unique neoplastic processes. Taking advantage of the unique concurrent methylation profile of multiple genes in cholangiocarcinoma may facilitate the distinction of cholangiocarcinoma from benign biliary epithelium in clinical settings.
\end{abstract}

Modern Pathology (2005) 18, 412-420, advance online publication, 1 October 2004; doi:10.1038/modpathol.3800287

Keywords: cholangiocarcinoma; tumor suppressor gene; DNA methylation; epigenetic

Cholangiocarcinoma is an uncommon but highly aggressive malignancy, which arises from the biliary ductal epithelium, either intrahepatically or extrahepatically. There is evidence that the neoplastic transformation of biliary epithelial cells and malignant progression of cholangiocarcinoma is accompanied by a number of genetic and epigenetic

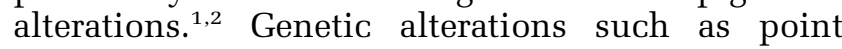
mutations of $K$-ras and p53 have been found in a subset of cholangiocarcinoma. ${ }^{3-6}$ Mutation or dele-

Correspondence: Dr B Yang, MD, PhD, Department of Anatomic Pathology, L25 Cleveland Clinic Foundation, 9500 Euclid Avenue, Cleveland, OH 44195, USA.

E-mail: yangb@ccf.org

Received 28 May 2004; revised 15 July 2004; accepted 26 July 2004; published online 1 October 2004 tion of $p 14^{A R F}$ and $p 16^{I N K 4 a}$ were not frequent events in cholangiocarcinoma. ${ }^{7,8}$ Although overexpression of $\beta$-catenin was frequently encountered in cholangiocarcinoma, mutation of $\beta$-catenin has not been identified to date. ${ }^{9}$ These studies indicate that genetic alterations frequently seen in other epithelial cancers are not commonly seen in cholangiocarcinoma.

Recent studies have shown that the function of a tumor suppressor gene can be silenced through promoter methylation. Epigenetic inactivation of a set of tumor suppressor genes through promoter methylation had been recently studied in intrahepatic cholangiocarcinoma in Asian populations. ${ }^{2}$ However, to our knowledge, methylation profiles of extrahepatic cholangiocarcinoma has not been well characterized. To further understand the epigenetic 
changes in cholangiocarcinoma and to see the difference in epigenetic alterations between intrahepatic cholangiocarcinoma and extrahepatic cholangiocarcinoma, we have studied the promoter methylation profiles of 12 candidate tumor suppressor genes in 72 cases of cholangiocarcinoma, including 36 cases of intrahepatic cholangiocarcinoma and 36 cases of extrahepatic cholangiocarcinoma and compared to 10 cases of benign biliary epithelia. In all, 12 tumor suppressor genes, involved in several signal transductional pathways, are adenomatous polyposis coli (APC), E-cadherin/CDH1, $O^{6}$-methylguanine methyltransferase (MGMT), rasassociated secreted factor (RASSF1A), glutathione $S$-transferase (GSTP), retinoic acid receptor beta-2 $(R A R-\beta), p 14^{A R F}, p 15^{I N K 4 B}, p 16^{I N K 4 A}, p 73, h M L H 1$ and death-associated protein kinase ( $D A P K)$.

\section{Materials and methods}

\section{Tumor Samples}

In all, 72 cases of cholangiocarcinoma, including 36 cases of intrahepatic cholangiocarcinoma and 36 cases of extrahepatic cholangiocarcinoma, were collected from Johns Hopkins Pathology and Cytopathology Archives, with the permission of Johns Hopkins University Institutional Review Board. The specimens include 52 cases of paraffin-embedded surgical resection specimens and 20 cases of cytologic smears from fine-needle aspiration biopsies. All the cytologic cases had confirmed diagnosis from subsequent tumor biopsies or resections. In all, 10 cases of negative bile duct surgical margins were used as control. No initial chemotherapy or radiation therapy was instituted before tumor excision or fineneedle aspiration and biopsy procedure. The tissue was fixed either in buffered-formalin for surgical specimens or in ethanol-based fixative for fine-needle aspiration and biopsy specimens. Consecutive sections were cut at $4 \mu \mathrm{m}$ from paraffin-embedded tissue blocks and mounted for histopathologic evaluation using conventional hematoxylin and eosin (H\&E) staining. H\&E-stained sections also served as a guide for DNA analysis. For DNA extraction, consecutive $10 \mu \mathrm{m}$ sections from resected specimens were directly collected into sterile Eppendorff tubes. Cells from cytologic smears were scraped into Eppendorff tubes by sterile surgical blades. Genomic DNA was isolated by digestion with $100 \mu \mathrm{g} / \mathrm{ml}$ proteinase $\mathrm{K}$ and followed by conventional phenol/chloroform (1:1) extraction and ethanol precipitation.

\section{Bisulfite Modification and Methylation-Specific PCR}

Bisulfite modification and methylation-specific PCR were conducted based on the principle that bisulfite treatment of DNA converts unmethylated cytosine residues into uracil, whereas methylated cytosine residues remain unmodified. Thus, after bisulfite conversion, methylated and unmethylated DNA sequences can be distinguished by sequence-specific primers. DNA was treated with sodium bisulfite as previously described. ${ }^{10}$ Briefly, $1 \mu \mathrm{g}$ of genomic DNA was denatured by incubation with $0.2 \mathrm{M} \mathrm{NaOH}$ for $10 \mathrm{~min}$ at $37^{\circ} \mathrm{C}$. Aliquots of $10 \mathrm{mM}$ hydroquinone (30 $\mu$ l; Sigma Chemical Co., St Louis, MO, USA) and $3 \mathrm{M}$ sodium bisulfite (pH 5.0, $520 \mu \mathrm{l}$; Sigma Chemical Co.) were added and the solution was incubated at $50^{\circ} \mathrm{C}$ for $16 \mathrm{~h}$. Treated DNA was purified by use of Wizard DNA purification System (Promega Corp., Madison, WI, USA), desulfonated with 0.3 $\mathrm{M} \mathrm{NaOH}$, precipitated with ethanol, and resuspended in water. Modified DNA was stored at $-70^{\circ} \mathrm{C}$ until used. DNA sequences containing promoter regions of $A P C$, E-cadherin/CDH1, RASSF1A, GSTP, RAR- $\beta, p 14^{A R F}$, $p 15^{I N K 4 b}, p 16^{I N K 4 a}, p 73$, MGMT, hMHL1 and DAPK genes were first amplified in a single PCR run with 30 cycles using flanking primer sets as previously described. ${ }^{11,12}$ DNA methylation of CpG islands was then determined by PCR using specific primers for both methylated and unmethylated DNA. ${ }^{11,12}$ Two sets of primers were used to amplify each region of interest: one pair recognized a sequence in which CpG sites are unmethylated (bisulfite modified to UpG), and the other recognized a sequence in which CpG sites are methylated (unmodified by bisulfite treatment). Negative control samples without DNA template (water only) and DNA were included for each set of PCR. PCR products were analyzed on 1\% polyacrylamide gels. Methylation status of $h M H L-1$ gene was analyzed only in 52 cases of surgical specimens; however not in cytologic specimens, due to the limitation of samples in the latter. Methylation-specific PCR of all the cases with negative methylation status and some of the methylationpositive cases were repeated to confirm the results.

\section{Statistical Analysis}

$\chi^{2}$ - or Fisher exact tests, depending on the absolute numbers included in the analysis, were used to analyze the frequency and association of concurrent tumor suppressor gene methylation in cholangiocarcinoma. $\chi^{2}$ - or Fisher's exact test were also applied to the correlation between tumor suppressor gene methylation profiles and clinicopathologic data. The strength of bivariate association between pairs of tumor suppressor genes was calculated using Pearson's correlation coefficients. A logistic regression analysis was used to analyze the correlation between tumor suppressor gene methylation profiles and the degree of tumor differentiation.

\section{Results}

\section{Frequency of Tumor Suppressor Gene Promoter Methylation in Cholangiocarcinoma}

Promoter methylation profiles of 12 tumor suppressor gene from 72 cases of cholangiocarcinoma and 
10 cases of benign biliary epithelia is illustrated in Figure 1. The frequency of methylation of individual tumor suppressor gene is summarized in Table 1. Of 72 cases of cholangiocarcinoma, 61 cases (85\%) had methylation of at least one tumor suppressor gene, while 11 cases (15\%) had no detectable methylation of any 12 tumor suppressor genes studied. The frequency of tumor suppressor gene methylation in cholangiocarcinoma was: RASSF1A (65\%), $p 15^{I N K 4 b}$ (50\%), p16 $6^{I N K 4 a}(50 \%)$, APC (46\%), E-cadherin/ CDH1 (43\%), p14 $14^{A R F}$ (38\%), p73 (36\%), MGMT (33\%), hMHL-1 (25\%), GSTP (14\%), RAR- $\beta$ (14\%) and $D A P K(3 \%)$. In benign biliary epithelial tissues, methylation of tumor suppressor genes were either nondetectable $\left(p 14^{A R F}, p 73, M G M T, h M L H 1, E-\right.$ cadherin/CDH1, GSTP, RAR- $\beta$ and DAPK) or with low frequency $(20 \%$ for $R A S S F 1 A$ and $10 \%$ for $p 15^{I N K 4 b}, p 16^{I N K 4 a}$ and $\left.A P C\right)$. There was a statistically significant difference between cholangiocarcinoma and benign biliary tissue in the frequency of methylation of RASSF1A, p16 $6^{I N K 4 a}, p 15^{I N K 4 b}, A P C$, $p 14^{A R F}$ and $p 73$ (Fisher's exact test, $P=0.012,0.017$, $0.020,0.020,0.026$ and 0.026 , respectively). There were marginal differences between cholangiocarcinoma and benign biliary epithelium in methylation of MGMT and E-cadherin/CDH1 $(P=0.056$ and 0.078 , respectively).

\section{Concurrent Methylation of Multiple Tumor Suppressor Genes in Cholangiocarcinoma}

Promoter methylation of multiple tumor suppressor genes was frequently seen in cholangiocarcinoma. Number of tumor suppressor genes methylated ranged from one to nine genes in cholangiocarcinoma. Although methylation of single tumor suppressor gene was seen in $50 \%(5 / 10)$ of cases of benign biliary epithelia, it was only encountered in two cases $(3 \%)$ of cholangiocarcinoma. Of 72 cases of cholangiocarcinoma, 59 cases $(82 \%)$ had methylation of two or more tumor suppressor genes (Table 2). In contrast, methylation of two or more tumor suppressor gene promoters was not seen in benign biliary tissue (Figure 1). Furthermore, 50 cases $(70 \%)$ of cholangiocarcinoma had methylation of three or more tumor suppressor genes and 37 cases (52\%) of cholangiocarcinoma had methylation of four or more tumor suppressor genes (Table 2). Methylation of multiple tumor suppressor genes tends to occur in those most frequently methylated genes, such as RASSF1, APC, $p 15^{I N K 4 b}$ and $p 16^{I N K 4 a}$. For instance, concurrent methylation of four or more tumor suppressor gene was seen in about $66 \%$ (31/47) cases of cholangiocarcinoma that harbored RASSF1A methylation; however, was found only in
$32 \%(8 / 25)$ of cases without RASSF1A methylation. The difference between these two groups was statistically significant $(P=0.028)$. Similarly, there was also a close correlation between methylation of hMLH1 promoter and concurrent methylation of multiple tumor suppressor genes in cholangiocarcinoma. Of 52 cases of cholangiocarcinoma analyzed for $h M L H 1$, methylation of the $h M L H 1$ promoter was found in 25\% (13/52) cases. Among hMLH1 methylators, methylation of five or more tumor suppressor genes was seen in $92 \%(12 / 13)$ cases. In contrast, only $39 \%(15 / 39)$ cases without hMLH1 methylation had methylation of five or more tumor suppressor genes $(P=0.001$, Table 3$)$.

\section{Association between Methylation of Various Genes in Cholangiocarcinoma}

To see whether epigenetic inactivation of a restricted number of pathways or of numerous signaling pathways involved in development of cholangiocarcinoma, we analyzed the strength of bivariate association between pairs of tumor suppressor genes methylated in cholangiocarcinoma using Pearson's correlation coefficients. Overall, methylation of all 12 tumor suppressor genes, except DAPK, showed some degree of association with each other. In all, 20 pairs of tumor suppressor genes were identified with significant correlation $(P<0.01)$. Among these 20 pairs, 16 pairs associated with methylation of three core genes: RASSF1A, $p 16^{I N K 4 a}$ and $h M L H 1$ (Figure 2). Not only was methylation of these three tumor suppressor genes correlated each other, but also methylation of each of these three tumor suppressor genes (RASSF1A, p16 $6^{I N K 4 a}$ and hMLH1) correlated significantly $(P<0.01)$ with methylation of other tumor suppressor genes (Figure 2a). Specifically, methylation of RASSF1A promoter was closely associated with the methylation of GSTP, $h M L H 1, p 14^{A R F}, p 16^{I N K 4 a}$ and $p 73$. Methylation of $p 16^{I N K 4 a}$ promoter was significantly associated with the methylation of $A P C, E$-cadherin/CDH1, hMLH1, $p 14^{A R F}, p 15, p 73, R A R-\beta$ and RASSF1A. Whereas methylation of $h M L H 1$ promoter was strongly associated with the methylation of MGMT, $p 14^{A R F}$, $p 16^{I N K 4 a}, R A R-\beta$ and RASSF1A (Figure 2a).

\section{Methylation Profiles between Intrahepatic Cholangiocarcinoma and Extrahepatic Cholangiocarcinoma}

Promoter methylation profiles of tumor suppressor genes were compared between 36 cases of intrahepatic cholangiocarcinoma and 36 cases of extrahepatic cholangiocarcinoma. In terms of methylation

Figure 1 Promoter methylation profiles of 12 candidate tumor suppressor genes in intrahepatic cholangiocarcinoma, extrahepatic cholangiocarcinoma and benign biliary epithelia. Blank space represents no detectable promoter methylation of an individual tumor suppressor gene and black space represents detectable promoter methylation of an individual tumor suppressor gene. Space with cross represents the case that has not been analyzed for hMHL1. 


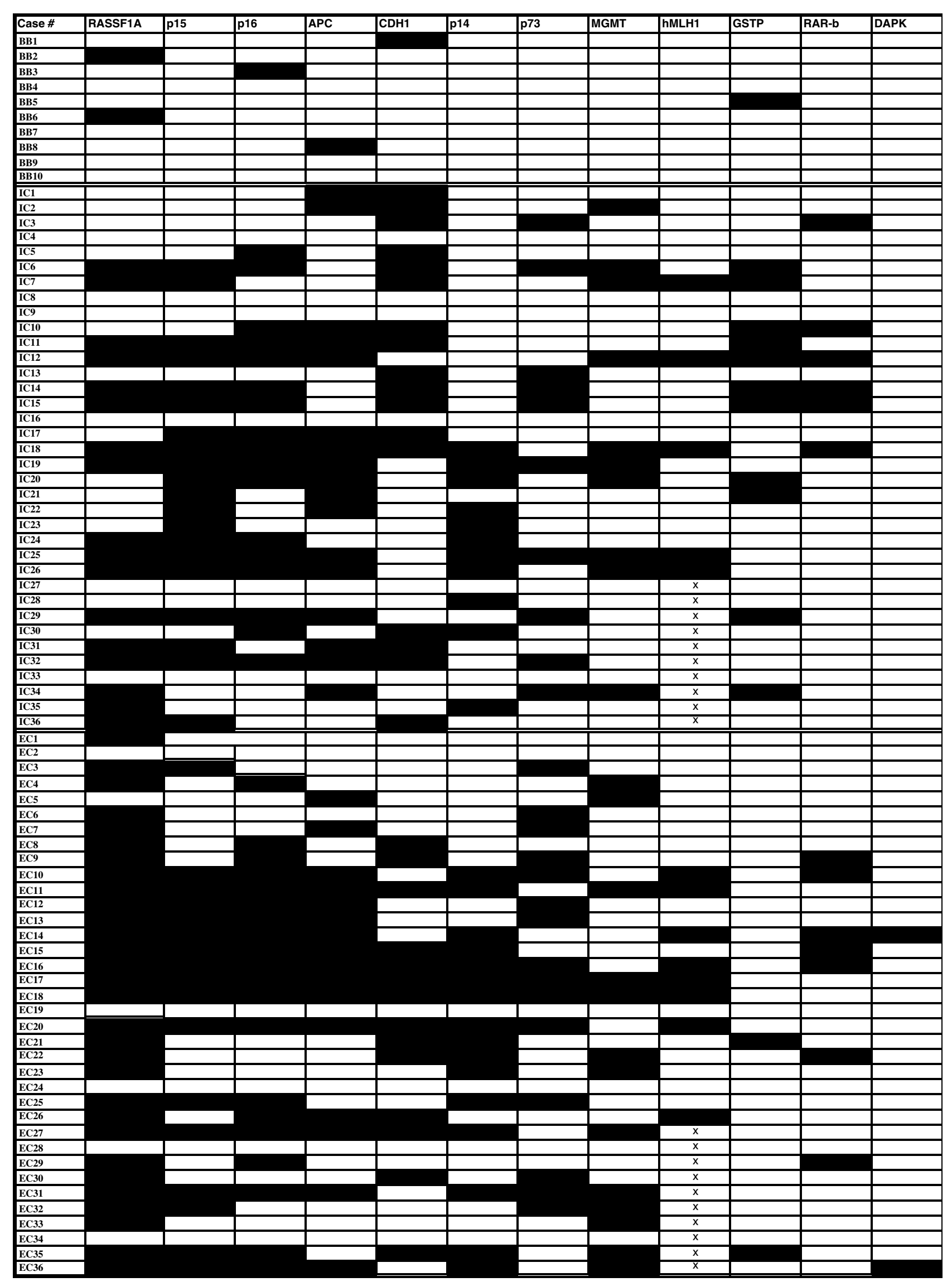


Table 1 Frequency of methylation of tumor suppressor genes in cholangiocarcinoma

\begin{tabular}{|c|c|c|c|c|c|c|c|c|c|c|c|c|}
\hline & RASSF-1 & p15 & p16 & $A P C$ & $E$-cad & p14 & $p 73$ & $M G M T$ & $\mathrm{hMLH}^{\mathrm{a}}$ & GSTP & $R A R-\beta$ & $D A P K$ \\
\hline CC & $\begin{array}{c}65.3 \% \\
(47)\end{array}$ & $\begin{array}{c}51.4 \% \\
(37)\end{array}$ & $\begin{array}{c}50.0 \% \\
(36)\end{array}$ & $\begin{array}{c}45.8 \% \\
(33)\end{array}$ & $\begin{array}{c}43.1 \% \\
(31)\end{array}$ & $\begin{array}{c}37.5 \% \\
(27)\end{array}$ & $\begin{array}{c}36.1 \% \\
(26)\end{array}$ & $\begin{array}{c}33.0 \% \\
(24)\end{array}$ & $\begin{array}{c}23.6 \% \\
(13)\end{array}$ & $\begin{array}{c}18.1 \% \\
(13)\end{array}$ & $\begin{array}{c}18.1 \% \\
(13)\end{array}$ & $\begin{array}{c}2.7 \% \\
\text { (2) }\end{array}$ \\
\hline ICC & $\begin{array}{c}48.6 \% \\
(17)\end{array}$ & $\begin{array}{c}54.1 \% \\
(20)\end{array}$ & $\begin{array}{c}48.6 \% \\
(17)\end{array}$ & $\begin{array}{c}47.2 \% \\
(17)\end{array}$ & $\begin{array}{c}48.6 \% \\
(17)\end{array}$ & $\begin{array}{c}30.0 \% \\
(11)\end{array}$ & $\begin{array}{c}27.0 \% \\
(10)\end{array}$ & $\begin{array}{c}27.0 \% \\
(10)\end{array}$ & $\begin{array}{c}18.5 \% \\
(5)\end{array}$ & $\begin{array}{c}31.4 \% \\
(11)\end{array}$ & $\begin{array}{c}16.0 \% \\
(6)\end{array}$ & $\begin{array}{c}0 \% \\
(0)\end{array}$ \\
\hline ECC & $\begin{array}{c}83.3 \% \\
(30)\end{array}$ & $\begin{array}{c}48.6 \% \\
(17)\end{array}$ & $\begin{array}{c}54.3 \% \\
(19)\end{array}$ & $\begin{array}{c}44.4 \% \\
(16)\end{array}$ & $\begin{array}{c}40.0 \% \\
(14)\end{array}$ & $\begin{array}{c}46.0 \% \\
(16)\end{array}$ & $\begin{array}{c}43.0 \% \\
(15)\end{array}$ & $\begin{array}{c}40.0 \% \\
(14)\end{array}$ & $\begin{array}{c}32.0 \% \\
(8)\end{array}$ & $\begin{array}{c}5.7 \% \\
(2)\end{array}$ & $\begin{array}{c}20.0 \% \\
(7)\end{array}$ & $\begin{array}{c}5.7 \% \\
(2)\end{array}$ \\
\hline
\end{tabular}

CC—cholangiocarcinoma; ICC-intrahepatic cholangiocarcinoma; ECC-extrahepatic cholangiocarcinoma.

${ }^{\mathrm{a}}$ Total 55 cases were used for $h M L H$ MSP analysis.

Table 2 Concurrent methylation of tumor suppressor genes in cholangiocarcinoma

\begin{tabular}{lccc}
\hline Number of TSG & CC (\%) (72) & ICC (\%) (36) & ECC (\%) (36) \\
\hline 0 TSG & $15.3(11)$ & $16.7(6)$ & $13.9(5)$ \\
1 TSG & $2.7(2)$ & $2.7(1)$ & $2.7(1)$ \\
2 TSGs & $11.2(8)$ & $13.9(5)$ & $8.3(3)$ \\
3 TSGs & $19.4(14)$ & $16.7(6)$ & $22.2(8)$ \\
$\geq 4$ TSGs & $51.4(37)$ & $50.0(18)$ & $52.9(19)$ \\
\hline
\end{tabular}

Absolute case numbers are included within parentheses. Abbreviations: TSG—-tumor suppressor gene; CC—cholangiocarcinoma; ICCintrahepatic cholangiocarcinoma; ECC-extrahepatic cholangiocarcinoma.

Table 3 Association of methylation status of hMLH1 with concurrent methylation index of multiple tumor suppressor genes in cholangiocarcinoma

\begin{tabular}{lccc}
\hline CMI & $\begin{array}{c}\text { hMLH1 } \\
\text { methylator (\%) } \\
(\mathrm{n}=13)\end{array}$ & $\begin{array}{c}\text { hMLH1 } \\
\text { nonmethylator (\%) } \\
(\mathrm{n}=39)\end{array}$ & P-values \\
\hline 0 TSGs & $0(0)$ & $17.9(7)$ & 0.172 \\
5 5 TSGs & $7.7(1)$ & $59.0(17)$ & 0.003 \\
$>$ 5 TSGs & $92.3(12)$ & $23.1(9)$ & 0.001 \\
\hline
\end{tabular}

CMI—concerted methylation index; TSG—-tumor suppressor gene.

frequency, methylation of $R A S S F 1 A$ was seen more frequently in extrahepatic cholangiocarcinoma (30/ 36) than that in intrahepatic cholangiocarcinoma (17/36) (83 vs $47 \%, P=0.003$ ). Although methylation of $p 14^{A R F}, p 73, M G M T$ and $h M L H 1$ was seen more often in extrahepatic cholangiocarcinoma than intrahepatic cholangiocarcinoma, the difference was not statistically significant $(P>0.05)$. On the other hand, methylation of GSTP promoter was more common in intrahepatic cholangiocarcinoma than in extrahepatic cholangiocarcinoma and this difference was statistically significant (31 vs $6 \%, P=0.012$ ). Although there was no significant difference in concurrent methylation of multiple tumor suppressor genes between intrahepatic and extrahepatic cholangiocarcinoma (Table 2), different bivariate association of pairs of tumor suppressor genes exists between intrahepatic cholangiocarcinoma and extrahepatic cholangiocarcinoma (Figure 2b and c). For example, methylation of GSTP or MGMT was not associated with methylation of other tumor suppressor genes in intrahepatic cholangiocarcinoma (Figure 2b), but methylation of GSTP was strongly $(P<0.01)$ associated with methylation of $p 15^{I N K 4 b}, p 16^{I N K 4 a}, p 73$ and $R A R-\beta$ in extrahepatic cholangiocarcinoma. Similarly, methylation of $M G M T$ was significantly $(P<0.05)$ associated with methylation of $A P C, h M L H 1$ and RASSF1A in extrahepatic cholangiocarcinoma (Figure 2c).

\section{Correlation of Tumor Suppressor Gene Methylation and Clinicopathologic Data}

Methylation profiles have been correlated with clinicopathologic data, such as patient gender, tumor size and tumor differentiation. There was no significant difference in the frequency of tumor suppressor gene methylation between genders (50 males and 22 females, $P>0.05$ ). The tumor size of cholangiocarcinoma ranged from 1.0 to $13 \mathrm{~cm}$. Tumor differentiation of 52 cases of surgical specimens was graded histopathologically into well-, moderately- and poorly differentiated. For intrahepatic cholangiocarcinoma cases, 26 cases include eight cases of well differentiated, 12 cases of moderately differentiated and six cases of poorly differentiated. For extrahepatic cholangiocarcinoma, 26 cases consist of 13 cases of well differentiated, nine cases of moderately differentiated and four cases of poorly differentiated. All the cases of intrahepatic cholangiocarcinoma and extrahepatic cholangiocarcinoma are classified as adenocarcinoma, not otherwise specified. There was no statistical significance between the frequency of individual tumor suppressor gene methylation and the degree of tumor differentiation ( $P>0.05$, data not shown). In terms of concerted multiple tumor suppressor gene methylation, methylation of four and more tumor suppression gene promoters was seen relatively more frequently in poorly differentiated tumors $(70 \%, 7 / 10)$ than moderately differentiated $(47 \%, 10 / 21)$ and well-differentiated tumors $(38 \%$, $8 / 21$ ). However, differences were not statistically significant between poorly- and moderately differentiated $(P=0.28)$ and between poorly- and welldifferentiated $(P=0.15)$. 
a APC

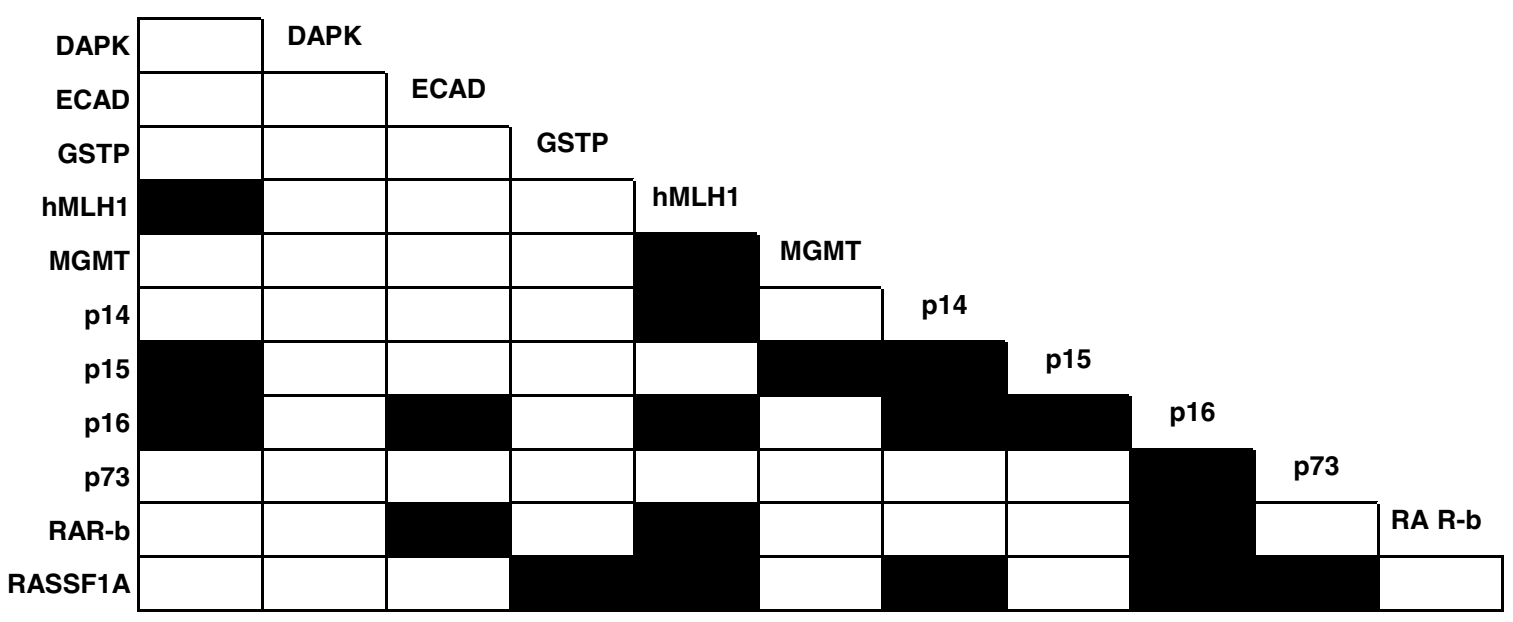

b

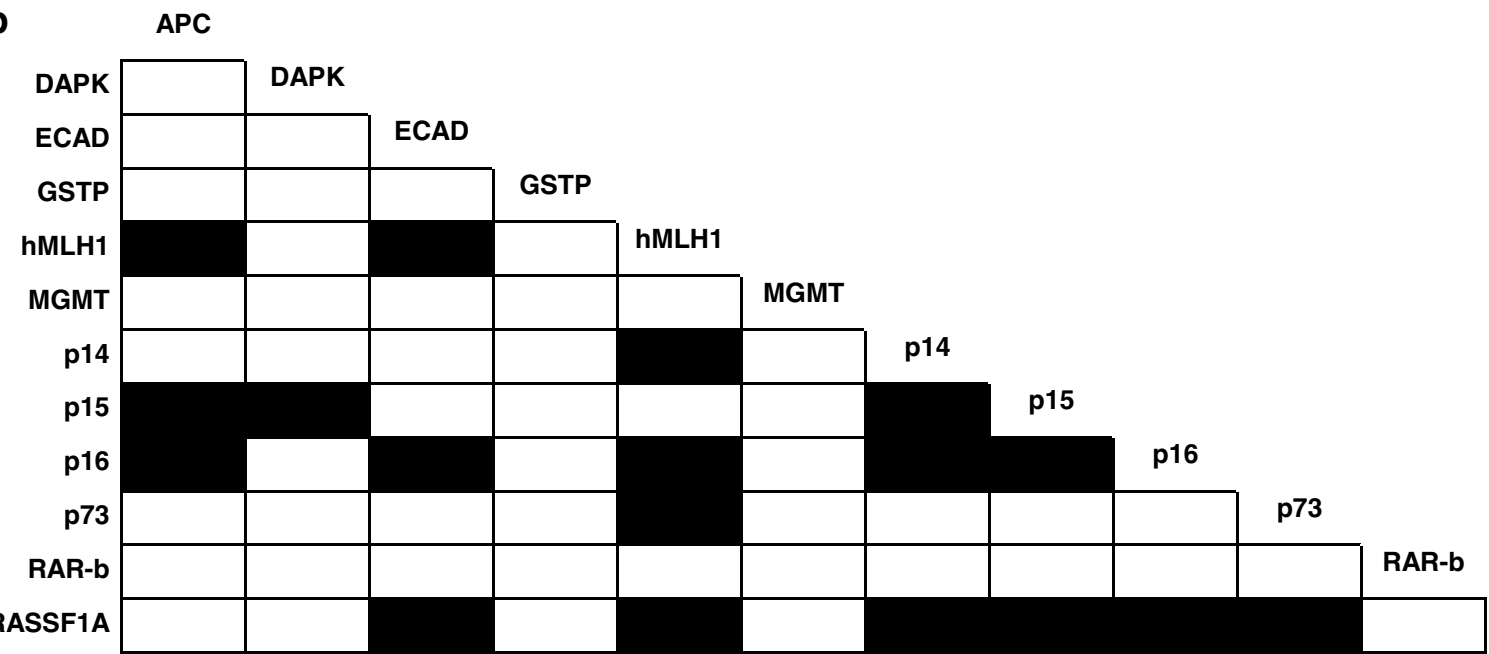

C APC

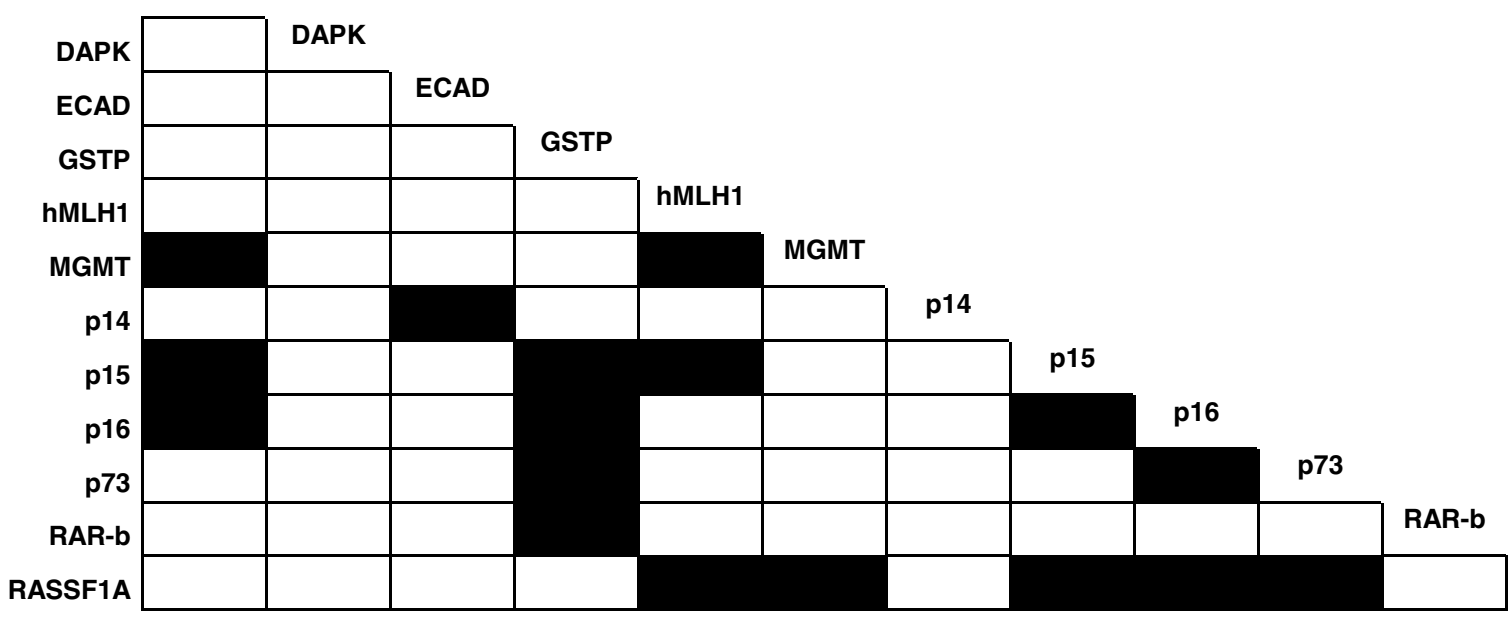

Figure 2 Many genes are coordinately methylated in cholangiocarcinoma. The strength of bivariate association between pairs of genes was calculated using Pearson's correlation coefficients. Black space represents methylation of pair of genes that demonstrates significant association $(P<0.01)$. (a) Bivariate association of promoter methylation of 12 tumor suppressor genes in all 72 cases of cholangiocarcinoma. (b) Bivariate association of methylation of tumor suppressor genes in 36 cases of extrahepatic cholangiocarcinoma. (c) Bivariate association of methylation of tumor suppressor genes in 36 cases of intrahepatic cholangiocarcinoma. 


\section{Discussion}

The molecular mechanisms of cholangiocarcinoma are largely unknown. Aberrant methylation of promoter $\mathrm{CpG}$ islands associated with human tumor suppressor genes has been studied as an alternative mechanism of gene inactivation that contributes to the biology of several human neoplasms. ${ }^{13,14}$ Although epigenetic changes of intrahepatic cholangiocarcinoma had been recently studied in Asian populations, ${ }^{2}$ methylation profiles of extrahepatic cholangiocarcinoma has not been reported to our knowledge. To further understand the epigenetic alterations in cholangiocarcinoma and to compare the methylation profiles between intrahepatic and extrahepatic cholangiocarcinoma, we have studied the methylation status of the promoter CpG islands of 12 tumor suppressor genes in 72 cases of cholangiocarcinoma, including 36 cases of intrahepatic cholangiocarcinoma and 36 cases of extrahepatic cholangiocarcinoma.

Our study demonstrates that epigenetic alteration of tumor suppressor genes, namely promoter hypermethylation, is a frequent event in cholangiocarcinoma. About $85 \%$ of cholangiocarcinoma had methylation of at least one tumor suppressor gene that we studied. Although methylation of each of 12 tumor suppressor gene promoters was seen in cholangiocarcinoma, the most frequently methylated tumor suppressor genes were RASSF1A (65\%), p15 $5^{I N K 4 b}(50 \%), p 16^{I N K 4 a}(50 \%), A P C(46 \%)$ and E-cadherin/CDH1 (43\%). One of the important findings from our study is that cholangiocarcinoma often had concurrent methylation of multiple tumor suppressor genes. Almost all methylated cases, except two, had methylation of two or more tumor suppressor genes. More than two-thirds of cholangiocarcinomas harbored methylation of three or more tumor suppressor gene promoters and more than half of the cases have methylation of four or more tumor suppressor gene promoters. It appears that development of cholangiocarcinoma may result from disruption of multiple signaling transduction pathways, such as the ras pathway (RASSF1A), Wnt pathway ( $A P C$ and E-cadherin/CDH1), RB pathway $\left(p 16^{I N K 4 a}\right)$, cell cycle control ( $\left.p 15^{I N K 4 b}\right), p 53$ pathway ( $p 14^{A R F}$ and $p 73$ ) and microsatellite stability (hMLH1). Our data further indicate that accumulation of such epigenetic alterations through concerted methylation of multiple tumor suppressor genes is required during carcinogenesis of the biliary epithelium.

As 12 tumor suppressor genes examined here represents important elements of several signaling pathways, we are interested to see whether epigenetic inactivation of these tumor suppressor genes reflects disruption of either restricted or numerous signaling pathways involved in the development of cholangiocarcinoma and whether there are linkages between different pathways. Through the analysis of the strength of bivariate association between pairs of tumor suppressor genes methylated in cholangiocarcinoma, we found 20 pairs of tumor suppressor genes with significant correlation coefficients $(P<0.01)$. Interestingly, 16 out of 20 pairs of such association are strongly correlated with the methylation of three core tumor suppressor genes: RASS1A, p16 and hMLH1, which are involved in three separate signaling pathways (ras pathway, pRb pathway and DNA mismatch repair pathway). Our data suggest that these three signaling pathways are likely the main targets epigenetically altered during the development of cholangiocarcinoma.

The human ras association domain family RASSF1A gene was cloned from the lung tumor suppressor locus $3 \mathrm{p} 21.3 .^{15}$ It has been shown that the expression of RASSF1A can be silenced through promoter methylation. Indeed, epigenetic inactivation is probably the main method that inactivates RASSF1A in cancer cells, since mutation of RASSF1A is extremely rare in cancer cells. ${ }^{16}$ Methylation of RASSF1A has been found in lung, breast, ovarian, renal cell, bladder and gastric carcinomas. ${ }^{17-22}$ We found that the vast majority cases of cholangiocarcinoma had methylation of RASSF1A promoter. Methylation of RASSF1A is often associated with concurrent methylation of multiple other tumor suppressor genes. The latter implies that methylation of RASSF1A may be one of the early events during biliary epithelial carcinogenesis. Although the biologic function of RASSF1A is still not entirely clear, recent studies indicate that RASSF $1 A$ is an important effector in mediating rasinduced apoptosis. ${ }^{23-26}$ It has been long speculated that the ras pathway is one of the major targets altered during biliary epithelial carcinogenesis. Previous genetic studies have shown that activation of the ras oncogene through point mutations presented in a subset of cholangiocarcinoma, particularly in perihilar cholangiocarcinoma., ${ }^{427}$ Our study further suggests that disruption of the ras pathway through the methylation of CpG islands in the $R A S S F 1 A$ promoter may also contribute to the development of cholangiocarcinoma. Recent studies indicate that methylation of RASSF1A and ras mutations are mutually exclusive in colorectal cancers. $^{28}$ Whether such phenomenon exists in cholangiocarcinoma needs further investigation.

Recent studies have indicated that the Wnt pathway is also involved in the pathogenesis of cholangiocarcinoma. Immunohistochemical study has shown that altered expression of $\beta$-catenin is found in up to $84 \%$ of cholangiocarcinoma. ${ }^{9}$ However, mutation of $\beta$-catenin was not yet identified by sequencing 58 cases of cholangiocarcinoma. ${ }^{9}$ Reduced expression of E-cadherin/CDH1 was also reported in cholangiocarcinoma. ${ }^{29}$ We found that about $69 \%$ of cases of cholangiocarcinoma had methylation of either $A P C$ or E-cadherin/CDH1 or both, including $46 \%$ of cholangiocarcinoma with methylation of $A P C$ and $43 \%$ cases with methylation of E-cadherin/CDH1. Our data suggest that 
promoter methylation of $A P C$ and/or E-cadherin/ $C D H 1$ may contribute in certain extent to the disruption of Wnt pathway and perhaps the activation of $\beta$-catenin in cholangiocarcinoma. Interestingly, methylation of $A P C$ and E-cadherin/CDH1 seems to be mutually exclusive in majority of cholangiocarcinoma. Approximately two-third of cases of cholangiocarcinoma had methylation of either $A P C$ or E-cadherin/CDH1; however, not both. A similar phenomenon was also observed in p53 pathway. We found that $68 \%$ of the cases had methylation of either $p 14^{A R F}$ or $p 73$. Our data suggest that disruption of one important element in the same signaling pathway is probably enough to diminish the function of the signaling pathway. According to Knudson's two-hit theory in cancer cells, it will be particularly interesting to see whether correlation between genetical loss of heterozygosity of one allele and epigenetic inactivation of remaining allele of such a frequently methylated tumor suppressor gene exists in cholangiocarcinoma.

Previous genetic studies have shown that there are different profiles between intrahepatic and extrahepatic cholangiocarcinoma. For instance, Kang et $\mathrm{al}^{3}$ found more frequent $K$-ras mutation, in cholangiocarcinoma arising in major bile ducts such as hilar cholangiocarcinoma, but only very rare mutations seen in peripheral type or intrahepatic cholangiocarcinoma. A recent immunohistochemical study also suggests that intrahepatic cholangiocarcinoma and extrahepatic cholangiocarcinoma have different cytokeratin immunoprofiles. ${ }^{30}$ Intrahepatic cholangiocarcinoma tends to be CK7 + /CK20-, while extrahepatic cholangiocarcinoma is more frequently CK7 $+/$ CK20.$+{ }^{30}$ To further understand the epigenetic changes in cholangiocarcinoma, we have compared the methylation profiles and patterns between intrahepatic- and extrahepatic cholangiocarcinoma. We found that different methylation profiles do exist between intrahepaticand extrahepatic cholangiocarcinoma. Most of the tumor suppressor genes analyzed had similar methylation frequencies between intrahepatic- and extrahepatic cholangiocarcinoma, such as methylations of $A P C, E$-cadherin/CDH1, p15 $5^{I N K 4 b}, p 16^{I N K 4 a}$ and MGMT. However, methylation of RASSF1A and GSTP showed significant differences between intrahepatic- and extrahepatic cholangiocarcinoma. Methylation of RASSF1A was more frequently seen in extrahepatic cholangiocarcinoma than in intrahepatic cholangiocarcinoma (84 vs 47\%), whereas methylation of GSTP was more commonly seen in intrahepatic cholangiocarcinoma (31 vs 6\%). The high frequency of RASSF1A methylation closely resembles that seen in adenocarcinoma of the pancreas. ${ }^{16}$ Whereas the higher frequency of GSTP methylation may be related to the close proximity between intrahepatic cholangiocarcinoma and hepatocellular carcinoma, which also harbors frequent methylation of GSTP promoter. ${ }^{31}$ By dissecting the association between pairs of tumor suppressor gene methylation (Figure 2a and b), we found that although six pairs of such associations (mainly with $R A S S F 1 A$ and $A P C$ ) were observed both in intrahepatic- and extrahepatic cholangiocarcinoma, majority of the bivariate associations were different between intrahepatic- and extrahepatic cholangiocarcinoma. Our findings support the notion that intrahepatic- and extrahepatic cholangiocarcinoma may be two closely related but different biologic entities.

Accurate diagnosis of cholangiocarcinoma in cytologic specimens, such as biliary brushing and fine-needle aspiration biopsy, is notoriously challenging for pathologists. One of the main reasons is that most cholangiocarcinomas have well-differentiated histopathologically and morphologically overlap with reactive biliary epithelia. As concurrent methylation of multiple tumor suppressor genes was not seen in benign biliary epithelium, methylationspecific PCR analysis of a set of tumor suppressor genes could be useful in facilitating accurate diagnosis of cholangiocarcinoma from benign/reactive biliary epithelium in preoperative biopsy or cytologic brushings. By analysis of a limited number of cytologic samples (biliary brushing and fineneedle aspiration and biopsy smears), we found that cytologic specimens (air-dried smears with DiffQuik stain or alcohol-fixed smears with Papanicolaou stain) yield equal or better methylation-specific PCR results compared to recuts from paraffinembedded tissues, probably due to less genomic fragmentation in cytologic specimens than formalinfixed surgical specimens. Besides its fast and less complicated technical merits, one of the advantages of methylation-specific PCR technique is that it can differentially detect hypermethylated allele (often seen in malignant cells) from unmethylated allele (often seen in normal cells) by designing different size of the PCR products. This is particularly useful for applying it in clinical materials since it does not require the enrichment of cancer cells from background reactive inflammatory cells and stromal cells. It should be noted that our comparison tissues were not normal bile ductal epithelia, but rather benign biliary epithelia of negative surgical margins that are far $(>2 \mathrm{~cm})$ from cancerous lesions. In order to assess the utility of methylation profiling in diagnostic pathology, it is important to study the background benign tissue surrounding malignant lesions. Our data show that although single gene methylation can be seen in benign biliary epithelia, it is the concurrent multiple gene methylation profiling that may be clinically useful in distinguishing cholangiocarcinoma from benign biliary epithelium.

In summary, we found that promoter methylation of tumor suppressor genes was frequent in cholangiocarcinoma. Intrahepatic- and extrahepatic cholangiocarcinoma have overlapping but different methylation profiles. Concurrent methylation of 
multiple tumor suppressor genes is a unique finding in cholangiocarcinoma since it was not found in benign biliary epithelia. Such concurrent methylation patterns in cholangiocarcinoma may be a useful adjunctive diagnostic test for cholangiocarcinoma in clinical samples, such as cytologic or small biopsy specimens that are not uncommonly frequent with difficulty using morphologic features alone.

\section{References}

1 Rashid A. Cellular and molecular biology of biliary tract cancers. Surg Oncol Clin N Am 2002;11: 995-1009.

2 Lee S, Kim WH, Jung HY et al. Aberrant CpG island methylation of multiple genes in intrahepatic cholangiocarcinoma. Am J Pathol 2002;161:1015-1022.

3 Kang YK, Kim WH, Lee HW et al. Mutation of p53 and K-ras, and loss of heterozygosity of APC in intrahepatic cholangiocarcinoma. Lab Invest 1999;79:477-483.

4 Sturm PD, Baas IO, Clement MJ et al. Alterations of the p53 tumor-suppressor gene and K-ras oncogene in perihilar cholangiocarcinomas from a high-incidence area. Int J Cancer 1998;78:695-698.

5 Kiba T, Tsuda H, Pairojkul C et al. Mutations of the p53 tumor suppressor gene and the ras gene family in intrahepatic cholangiocellular carcinomas in Japan and Thailand. Mol Carcinog 1993;8:312-318.

6 Wattanasirichaigoon S, Tasanakhajorn U, Jesadapatarakul S. The incidence of K-ras codon 12 mutations in cholangiocarcinoma detected by polymerase chain reaction technique. J Med Assoc Thai 1998;81: 316-323.

7 Tannapfel A, Benicke M, Katalinic A et al. Frequency of p16(INK4A) alterations and K-ras mutations in intrahepatic cholangiocarcinoma of the liver. Gut 2000;47:721-727.

8 Ahrendt SA, Eisenberger CF, Yip L et al. Chromosome 9p21 loss and p16 inactivation in primary sclerosing cholangitis-associated cholangiocarcinoma. J Surg Res 1999;84:88-93.

9 Sugimachi K, Taguchi K, Aishima S et al. Altered expression of beta-catenin without genetic mutation in intrahepatic cholangiocarcinoma. Mod Pathol 2001;14:900-905.

10 Herman JG, Graff JR, Myohanen S et al. Methylationspecific PCR: a novel PCR assay for methylation status of CpG islands. Proc Natl Acad Sci USA 1996; 93:9821-9826.

11 House MG, Guo M, Iacobuzio-Donahue $\mathrm{C}$ et al. Molecular progression of promoter methylation in intraductal papillary mucinous neoplasms (IPMN) of the pancreas. Carcinogenesis 2003;24:193-198.

12 House MG, Herman JG, Guo MZ et al. Aberrant hypermethylation of tumor suppressor genes in pancreatic endocrine neoplasms. Ann Surg 2003; 238:423-431 discussion 431-432.

13 Herman JG, Baylin SB. Promoter-region hypermethylation and gene silencing in human cancer. Curr Top Microbiol Immunol 2000;249:35-54.

14 Baylin SB, Herman JG. DNA hypermethylation in tumorigenesis: epigenetics joins genetics. Trends Genet 2000;16:168-174.
15 Dammann R, Li C, Yoon JH et al. Epigenetic inactivation of a RAS association domain family protein from the lung tumour suppressor locus 3p21.3. Nat Genet 2000;25:315-319.

16 Dammann R, Schagdarsurengin U, Liu L et al. Frequent RASSF1A promoter hypermethylation and K-ras mutations in pancreatic carcinoma. Oncogene 2003;22:3806-3812.

17 Byun DS, Lee MG, Chae KS et al. Frequent epigenetic inactivation of RASSF1A by aberrant promoter hypermethylation in human gastric adenocarcinoma. Cancer Res 2001;61:7034-7038.

18 Honorio S, Agathanggelou A, Wernert N et al. Frequent epigenetic inactivation of the RASSF1A tumour suppressor gene in testicular tumours and distinct methylation profiles of seminoma and nonseminoma testicular germ cell tumours. Oncogene 2003;22: 461-466.

19 Morrissey C, Martinez A, Zatyka M et al. Epigenetic inactivation of the RASSF1A 3p213 tumor suppressor gene in both clear cell and papillary renal cell carcinoma. Cancer Res 2001;61:7277-7281.

20 Yoon JH, Dammann R, Pfeifer GP. Hypermethylation of the CpG island of the RASSF1A gene in ovarian and renal cell carcinomas. Int J Cancer 2001;94:212-217.

21 Lee MG, Kim HY, Byun DS et al. Frequent epigenetic inactivation of RASSF1A in human bladder carcinoma. Cancer Res 2001;61:6688-6692.

22 Agathanggelou A, Honorio S, Macartney DP et al. Methylation associated inactivation of RASSF1A from region 3p21.3 in lung, breast and ovarian tumours. Oncogene 2001;20:1509-1518.

23 Khokhlatchev A, Rabizadeh S, Xavier $\mathrm{R}$ et al. Identification of a novel Ras-regulated proapoptotic pathway. Curr Biol 2002;12:253-265.

24 Ortiz-Vega S, Khokhlatchev A, Nedwidek M et al. The putative tumor suppressor RASSF1A homodimerizes and heterodimerizes with the Ras-GTP binding protein Nore1. Oncogene 2002;21:1381-1390.

25 Vos MD, Ellis CA, Bell A et al. Ras uses the novel tumor suppressor RASSF1 as an effector to mediate apoptosis. J Biol Chem 2000;275:35669-35672.

26 Vos MD, Ellis CA, Elam C et al. RASSF2 is a novel K-Ras-specific effector and potential tumor suppressor. J Biol Chem 2003;278:28045-28051.

27 Isa $\mathrm{T}$, Tomita S, Nakachi A et al. Analysis of microsatellite instability, K-ras gene mutation and p53 protein overexpression in intrahepatic cholangiocarcinoma. Hepatogastroenterology 2002;49:604-608.

28 van Engeland M, Roemen GM, Brink M et al. K-ras mutations and RASSF1A promoter methylation in colorectal cancer. Oncogene 2002;21:3792-3795.

29 Asayama Y, Taguchi Ki K, Aishima Si S et al. The mode of tumour progression in combined hepatocellular carcinoma and cholangiocarcinoma: an immunohistochemical analysis of E-cadherin, alpha-catenin and beta-catenin. Liver 2002;22:43-50.

30 Rullier A, Le Bail B, Fawaz R et al. Cytokeratin 7 and 20 expression in cholangiocarcinomas varies along the biliary tract but still differs from that in colorectal carcinoma metastasis. Am J Surg Pathol 2000;24: 870-876.

31 Yang B, Guo M, Herman JG et al. Aberrant promoter methylation profiles of tumor suppressor genes in hepatocellular carcinoma. Am J Pathol 2003;163: 1101-1107. 